\title{
Distributed Interference-Aware Energy-Efficient Power Optimization
}

\author{
Guowang Miao, Nageen Himayat, Geoffrey Ye Li, and Shilpa Talwar
}

\begin{abstract}
Power optimization techniques are becoming increasingly important in wireless system design since battery technology has not kept up with the demand of mobile devices. They are also critical to interference management in wireless systems because interference usually results from both aggressive spectral reuse and high power transmission and severely limits system performance. In this paper, we develop an energy-efficient power optimization scheme for interference-limited wireless communications. We consider both circuit and transmission powers and focus on energy efficiency over throughput. We first investigate a non-cooperative game for energy-efficient power optimization in frequency-selective channels and reveal the conditions of the existence and uniqueness of the equilibrium for this game. Most importantly, we discover a sufficient condition for generic multichannel power control to have a unique equilibrium in frequencyselective channels. Then we study the tradeoff between energy efficiency and spectral efficiency and show by simulation results that the proposed scheme improves both energy efficiency and spectral efficiency in an interference-limited multi-cell cellular network.
\end{abstract}

Index Terms-Interference, energy efficiency, power optimization, OFDM, non-cooperative power control.

\section{INTRODUCTION}

A $\mathrm{S}$ more users need to share the same spectrum for wideband multimedia communications and cellular networks move towards aggressive full frequency reuse scenarios [1], the performance of wireless cellular networks is heavily impaired by interference. This motivates the use of multi-cell power control and optimization for interference management[1]-[4]. On the other hand, as higher capacity wireless links are designed to meet increasing demand from multimedia applications, the device power consumption also increases. In contrast, the improvement in battery technology is much slower, leading to an exponentially increasing gap between the required and available battery capacity[5]. Hence, power optimization is also important for maximizing the battery life for mobile devices. Although, power optimization plays a pivotal role in both interference management and

Manuscript received August 2, 2010; revised November 5, 2010; accepted January 10, 2011. The associate editor coordinating the review of this paper and approving it for publication was L. Deneire.

This work was supported by Intel Corp. and the U.S. Army Research Laboratory under the Collaborative Technology Alliance Program, Cooperative Agreement DAAD19-01-20-0011.

G. Miao was with the School of Electrical and Computer Engineering, Georgia Institute of Technology, Atlanta, Georgia, 30332. He is now with Dallas Telecom Lab, Samsung Telecom America (e-mail: guowangmiao@gmail.com).

G. Y. Li is with the School of Electrical and Computer Engineering, Georgia Institute of Technology, Atlanta, Georgia, 30332-0250.

N. Himayat and S. Talwar are with Communications Technology Lab., Intel Corporation, Santa Clara, CA.

Digital Object Identifier 10.1109/TWC.2011.021611.101376 energy utilization, little research has addressed their joint interaction. Only an implicit discussion can be found in [6], which summarizes existing approaches that addresses either throughput or energy efficiency separately in the context of power control for CDMA networks. In this paper, we will address this topic and develop energy-efficient power optimization, specifically for interference-limited environments.

Our previous work in [7]-[10] has studied energy efficiency issue in uplink communications in single-cell orthogonal frequency division multiple access (OFDMA) systems to improve mobile battery consumption, where both circuit and transmission powers have been considered. Using throughput per Joule as a performance metric, we have studied both link adaptation and resource allocation techniques. We have observed that in an interference free environment, a tradeoff between energy efficiency (EE) and spectral efficiency (SE) exists as increasing transmission power always improves throughput but not necessarily EE. In this paper, we consider multi-cell interference-limited scenarios and develop power optimization and resource allocation schemes to improve EE. We note that the general power optimization problem in the presence of interference is intractable even when ideal user cooperation is assumed. Therefore, we develop a non-cooperative game for energy-efficient power optimization. We show that the equilibrium always exists but may not always be unique, unless there is only one subchannel or the channel experiences flat fading. However, in frequency-selective channels, this is not true in general as demonstrated by a counter example. We reveal a sufficient condition that assures the uniqueness of the equilibrium. Then we investigate the tradeoff between $\mathrm{EE}$ and SE and show that in interference-limited scenarios, increased transmission power also brings higher interference to the network and SE is not necessarily increased. Due to the conservative nature of energy-efficient power allocation, interference from other cells is effectively controlled and network throughput can be improved. We also discuss the implementation of the non-cooperative power optimization game. Compared with existing power control schemes, the simulation results show that the proposed scheme improves both energy efficiency and spectral efficiency in an interference-limited cellular network.

The rest of the paper is organized as follows. We first formulate the multi-channel interference-aware power control problem in Section II. We then discuss a non-cooperative energy-efficient power optimization game and study the existence and uniqueness of the equilibrium for this game in Section III. In Section IV, we first investigate the equilibrium characteristics for general multichannel power control schemes 
and then give a sufficient condition that will assure the uniqueness of the equilibrium for the non-cooperative energyefficient power optimization game. In Section V, we study the tradeoff between spectral and energy efficiency. In Section VI, we develop a temporal iterative binary search algorithm for the non-cooperative power optimization game and the performance improvement is demonstrated by simulations in Section VII. Finally, we conclude the paper in Section VIII.

\section{Problem Description}

We introduce interference-aware energy-efficient power optimization in this section. Consider a system with $K$ subchannels. Each of them experiences independent and flat fading and additive white Gaussian noise (AWGN). There are $N$ users, each consisting of a pair of transmitter and receiver and operating on these subchannels. All users interfere with each other. Accurate channel state information is available to any pair of transmitter and receiver. Denote the signal power attenuation of User $i$ at subchannel $k$ to be $g_{i i}^{(k)}$ and the interference power gain from the transmitter of User $i$ to the receiver of User $j$ at subchannel $k$ to be $g_{i j}^{(k)}$. The noise power on each subchannel is $\sigma^{2}$. The power allocation of User $n$ on all subchannels is denoted by vector $\mathbf{p}_{n}=\left[p_{n}^{(1)} p_{n}^{(2)} \cdots p_{n}^{(K)}\right]$. The interference on all subchannels of User $n$ is denoted by vector $\mathbf{I}_{n}=\left[I_{n}^{(1)} I_{n}^{(2)} \cdots I_{n}^{(K)}\right]$, where

$$
I_{n}^{(k)}=\sum_{i=1, i \neq n}^{N} p_{i}^{(k)} g_{i n}^{(k)} .
$$

Consequently, the signal-to-interference-plus-noise ratio (SINR), $\eta_{n}^{(k)}$, of User $n$ at subchannel $k$ can be expressed as

$$
\eta_{n}^{(k)}=\frac{p_{n}^{(k)} g_{n n}^{(k)}}{\sum_{i=1, i \neq n}^{N} p_{i}^{(k)} g_{i n}^{(k)}+\sigma^{2}} .
$$

The data rate at subchannel $k$ of User $n, r_{n}^{(k)}$, is assumed to be a function of $\eta_{n}^{(k)}$ and can be expressed as

$$
r_{n}^{(k)}=R\left(\eta_{n}^{(k)}\right)
$$

where $R()$ is assumed to be strictly concave and increasing in SINR with $R(0)=0$. For capacity approaching coding [11], $r_{n}^{(k)}=w \log \left(1+\eta_{n}^{(k)}\right)$, where $w$ is the bandwidth of each subchannel.

Let the data rate vector of User $n$ across the $K$ subchannels be $\mathbf{r}_{n}=\left[r_{n}^{(1)}, r_{n}^{(2)}, \cdots, r_{n}^{(K)}\right]$, then the overall data rate is

$$
r_{n}=\sum_{k=1}^{K} r_{n}^{(k)} .
$$

The total transmission power is

$$
p_{n}=\sum_{k=1}^{K} p_{n}^{(k)} .
$$

Note that as in [7], [9], both transmission power and circuit power, $p_{c}$, are important for energy-efficient communications. While transmission power is used for reliable data transmission, circuit power represents average energy consumption of device electronics. As in [9], we optimize the energy efficiency, defined as

$$
u_{n}=\frac{r_{n}}{\triangle e / \triangle t}=\frac{r_{n}}{p_{n}+p_{c}},
$$

where $r_{n}$ is given by (4) and $p_{n}$ by (5). $u_{n}$ is called EE of User $n$.

If the overall transmission power is fixed, the objective of Equation (6) is equivalent to maximizing the overall throughput of all subchannels and existing water-filling power allocation approach [11] gives the solution. However, besides power distributions on all subchannels, the overall transmission power needs to be adapted according to the states of all subchannels to maximize energy efficiency. Hence, the solution to Equation (6) is in general different from the existing power allocation schemes that maximize throughput with power constraints. The power control in a multi-cell setting to optimize the overall network energy efficiency is also different from traditional power control schemes that emphasize throughput improvement.

The EE of the overall network can be defined as

$$
u=\sum_{n=1}^{N} u_{n}
$$

which is a function of $p_{n}^{(k)}$ for all $n$ and $k$. This definition is based on summation of EE of all users rather than the ratio of sum network throughput to sum network power consumption because powers of different users can not be shared and so are their throughput and EE.

We need to determine power allocation of all users to optimize the overall network EE subject to the interference scenario. Note that the solution maximizing sum network EE is difficult to obtain as the objective function is in general non-concave in $p_{n}^{(k)}$. More users and subchannels in the system will result in more local maximums and searching the globally optimal power allocation would be a daunting task. Even if the globally optimal solution can be found, it is still impractical since the central controller requires complete network knowledge, including interference channel gains. Hence, we consider distributed power optimization in this paper.

\section{Distributed ENERGY-EFFICIENT COMMUNiCATIONS}

In this section, we will discuss distributed energy-efficient communications, where no cooperation among users is assumed and all users apply the same policy using their own local information. In the following, we model the noncooperative energy-efficient power optimization from a gametheory perspective and then investigate the existence and uniqueness of its equilibrium.

\section{A. Non-cooperative Energy-Efficient Power Optimization Game}

Since the network energy efficiency depends on the behaviors of two or more users, we model the power control to be a non-cooperative game in game theory [12]. Rooted in economics, game theory has been broadly applied in wireless communications for random access and power control optimizations [2], [3]. 
Consider the power allocation of User $n$ and denote the power vectors of other users to be vector

$$
\mathbf{p}_{-n}=\left(\mathbf{p}_{1}, \mathbf{p}_{2}, \cdots, \mathbf{p}_{n-1}, \mathbf{p}_{n+1}, \cdots, \mathbf{p}_{N}\right) .
$$

Given the power allocation of all other users, $\mathbf{p}_{-n}$, the best response of the power allocation of User $n$ is given by

$$
\mathbf{p}_{n}^{o}=f_{n}\left(\mathbf{p}_{-n}\right)=\arg \max _{\mathbf{p}_{n}} u_{n}\left(\mathbf{p}_{n}, \mathbf{p}_{-n}\right),
$$

where $u_{n}$ is given by (6) and is a function of both $\mathbf{p}_{n}$ and $\mathbf{p}_{-n} . f_{n}\left(\mathbf{p}_{-n}\right)$ is called the best response function of User $n$. The existence and uniqueness of $\mathbf{p}_{n}^{o}$, i.e. the best response, is assured by Theorem I in our previous work [9], which is also summarized in Appendix B when we prove Theorem 1 of this paper.

Note that non-cooperative power control is not efficient in terms of SE optimization since users tend to act selfishly by increasing their transmission power beyond what is reasonable [2]. Hence, pricing mechanisms are introduced to regulate the aggressive power transmission by individuals to produce more socially beneficial outcome towards improving sum throughput of all users [3]. Different from SE optimal power control, energy-efficient power optimization desires a power setting that is greedy in EE but chary of power. Furthermore, Problem (9) is equivalent to

$$
\begin{aligned}
\mathbf{p}_{n}^{o} & =\arg \max _{\mathbf{p}_{n}} \log \left(u_{n}\left(\mathbf{p}_{n}, \mathbf{p}_{-n}\right)\right) \\
& =\arg \max _{\mathbf{p}_{n}}\left(\log \left(r_{n}\right)-\log \left(p_{n}+p_{c}\right)\right),
\end{aligned}
$$

which implies that energy-efficient power control can be regarded as a variation of traditional spectral-efficient one with power pricing [3]. Since this power-conservative expression is socially favorable in interference-limited scenarios, energyefficient power control is desirable to reduce interference and improve throughput in a non-cooperative setting.

Each user optimizes their power independently. The variation of power allocation of one user impacts those of all others. Equilibrium is the condition of a network in which competing influences are balanced assuming invariant channel conditions. Its properties are important to network performance. Hence, we characterize the equilibrium of non-cooperative energyefficient power optimization in the following three sections.

\section{B. Existence of Equilibrium}

In a non-cooperative game, a set of strategies is said to be at Nash equilibrium if no user can gain individually by unilaterally altering its own strategy. Denote the equilibrium as

$$
\mathbf{p}^{*}=\left(\mathbf{p}_{1}^{*}, \mathbf{p}_{2}^{*}, \cdots, \mathbf{p}_{N}^{*}\right) .
$$

Nash equilibrium can be strictly described by the following definition.

Definition 1. In an energy-efficient non-cooperative game, an equilibrium is a set of power allocation that no user can unilaterally improve its energy efficiency by choosing a different set of power allocation, i.e.

$$
\mathbf{p}^{*}=f\left(\mathbf{p}^{*}\right)=\left(f_{1}\left(\mathbf{p}_{-1}^{*}\right), f_{2}\left(\mathbf{p}_{-2}^{*}\right), \cdots, f_{N}\left(\mathbf{p}_{-N}^{*}\right)\right),
$$

where $f(\mathbf{p})$ is the network response function.
The network response relies on energy efficiency of all users. In the following, we first address the properties of energy efficiency function and then study the existence of equilibrium. To facilitate our discussion, we introduce the concept of quasiconcavity [13].

Definition 2. A function $z$, which maps from a convex set of real n-dimensional vectors, $\mathcal{D}$, to a real number, is called strictly quasiconcave if for any $\mathbf{x}_{1}, \mathbf{x}_{2} \in \mathcal{D}$ and $\mathbf{x}_{1} \neq \mathbf{x}_{2}$,

$$
z\left(\lambda \mathbf{x}_{1}+(1-\lambda) \mathbf{x}_{2}\right)>\min \left\{z\left(\mathbf{x}_{1}\right), z\left(\mathbf{x}_{2}\right)\right\},
$$

for any $0<\lambda<1$.

According to the above definition, the quasiconcavity of the energy efficiency function can be proved in Appendix A and is summarized in the following lemma.

Lemma 1. $u_{n}\left(\mathbf{p}_{n}, \mathbf{p}_{-n}\right)$ is strictly quasiconcave in $\mathbf{p}_{n}$.

Based on Lemma 1, the existence of the equilibrium $\mathbf{p}^{*}$ is given in Theorem 1. A necessary and sufficient condition for a set of power allocation to be an equilibrium is proved in in Appendix B and summarized in Theorem 1.

Theorem 1 (Existence). There exists at least one equilibrium $\mathrm{p}^{*}$ in the non-cooperative energy-efficient power optimization game defined by (9). A set of power allocation of all users, $\mathbf{p}^{*}=\left(\mathbf{p}_{1}^{*}, \mathbf{p}_{2}^{*}, \cdots, \mathbf{p}_{N}^{*}\right)$, is an equilibrium if and only if it satisfies that, for any subchannel $i$ of any User $n$,

(i) if $R^{\prime}(0) \gamma_{n}^{(i) *} \geq \frac{\sum_{j \neq i} r_{n}^{(j) *}}{p_{c}+\sum_{j \neq i} p_{n}^{(j) *}},\left.\frac{\partial u_{n}\left(\mathbf{p}_{n}, \mathbf{p}_{-n}^{*}\right)}{\partial p_{n}^{(i)}}\right|_{\mathbf{p}_{n}=\mathbf{p}_{n}^{*}}=0$, i.e. $R^{\prime}\left(\gamma_{n}^{(i) *} p_{n}^{(i) *}\right) \gamma_{n}^{(i) *}=u\left(\mathbf{p}_{n}^{*}, \mathbf{p}_{-n}^{*}\right)$;

(ii) otherwise, $p_{n}^{(i) *}=0$,

where $\gamma_{n}^{(i) *}=\frac{g_{n n}^{(i)}}{\sum_{j=1, j \neq n}^{N} p_{j}^{(i) *} g_{j n}^{(i)}+\sigma^{2}}$.

In $(i)$ of Theorem $1, \frac{\sum_{j \neq i} r_{n}^{(j) *}}{p_{c}+\sum_{j \neq i} p_{n}^{(j) *}}$ is the overall energy efficiency of User $n$ when subchannel $i$ is idle. Subchannel $i$ should be used only if using it can improve the overall energy efficiency of User $n$ and this is determined by how good the state of subchannel $i$ is. If $R^{\prime}(0) \gamma_{n}^{(i) *} \geq \frac{\sum_{j \neq i} r_{n}^{(j) *}}{p_{c}+\sum_{j \neq i} p_{n}^{(j) *}}$, which is equivalent to $g_{n n}^{(i)} \geq \frac{\sum_{j \neq i} r_{n}^{(j) *}}{p_{c}+\sum_{j \neq i} p_{n}^{(j) *}} \frac{\sum_{j=1, j \neq n}^{N} p_{j}^{(i) *} g_{j n}^{(i)}+\sigma^{2}}{R^{\prime}(0)}$, the state of subchannel $i$ is good enough for data transmission and the power allocated on it satisfies $R^{\prime}\left(\gamma_{n}^{(i) *} p_{n}^{(i) *}\right) \gamma_{n}^{(i) *}=$ $u\left(\mathbf{p}_{n}^{*}, \mathbf{p}_{-n}^{*}\right)$. When the power allocations of all users satisfy (i) or $(i i)$ in Theorem 1, the network is in an equilibrium state.

\section{Uniqueness of Equilibrium in Flat Fading Channels}

In this section, we discuss the uniqueness of the equilibrium. First, we consider a special case when there is a single subchannel in a network and

$$
p_{n}^{o}=f_{n}\left(\mathbf{p}_{-n}\right)=\arg \max _{p_{n}} u_{n}\left(p_{n}, \mathbf{p}_{-n}\right) .
$$

In this case, the properties of the response functions, proved in Appendix C, can be stated in Lemma 2.

Lemma 2. When there is only one subchannel, the power allocation, i.e. the response functions, of all users satisfy 
- Concavity: $f_{n}\left(\mathbf{p}_{-n}\right)$ is strictly concave in $\mathbf{p}_{-n}$;

- Positivity: $f_{n}\left(\mathbf{p}_{-n}\right)>0$;

- Monotonicity: If $\mathbf{p}_{-n} \succ \mathbf{q}_{-n}, f_{n}\left(\mathbf{p}_{-n}\right)>f_{n}\left(\mathbf{q}_{-n}\right)$;

- Scalability: For all $\alpha>1, \alpha f_{n}\left(\mathbf{p}_{-n}\right)>f_{n}\left(\alpha \mathbf{p}_{-n}\right)$,

where $\succ$ denotes vector inequality and each element of the vector satisfies the inequality.

Note that the monotonicity indicates that increasing interference results in increasing transmission power while the scalability indicates that variation of transmission power is always smaller than that of the interference power. These assure the convergence to a unique equilibrium.

The properties in Lemma 2 can be extended to networks with multiple subchannels where all subchannels experience the same channel gain, i.e. flat-fading channels. This can be done by defining $f_{n}\left(\mathbf{p}_{-n}\right)$ to be the optimal total transmission power on all subchannels and the four properties can be easily verified by the approaches in Appendix C.

Theorem 2 (Uniqueness). When the channel experiences flat fading, there exists one and only one equilibrium $\mathbf{p}^{*}$ in the non-cooperative energy-efficient power optimization game defined by (9).

Proof: It has been shown in [14] that a non-cooperative power control with positivity, monotonicity, and scalability has a unique fixed point $\mathbf{p}=f(\mathbf{p})$. Hence, we have the above theorem.

\section{Uniqueness of Equilibrium in Frequency-Selective Chan- nels}

When there are multiple subchannels that experience frequency-selective fading, whether there is a unique equilibrium depends on channel conditions.

As an example, consider a network with two users. Let $p_{c}=1, w=1, \sigma^{2}=1, g_{11}^{(1)}=g_{11}^{(2)}=g_{22}^{(1)}=g_{22}^{(2)}=1, g_{12}^{(1)}=$ $g_{21}^{(1)}=1 e^{-10}, g_{12}^{(2)}=g_{21}^{(2)}=1 e^{10}$. We show in Appendix $\mathrm{D}$ that one of the equilibrium has the form $\mathbf{p}_{1}^{*}=\left[\begin{array}{ll}p_{\alpha} & p_{\beta}\end{array}\right]$ and $\mathbf{p}_{2}^{*}=\left[p_{\gamma} 0\right]$, where $p_{\alpha}, p_{\beta}$, and $p_{\gamma}$ are positive. Due to the symmetry of network conditions, $\mathbf{p}_{1}=\left[\begin{array}{ll}p_{\gamma} & 0\end{array}\right]$ and $\mathbf{p}_{2}=\left[p_{\alpha} p_{\beta}\right]$ must be another equilibrium. Hence, the network has at least two equilibria. When there are more users and subchannels, more equilibria may exist. However, when the interfering channels satisfy a certain condition, there will be a unique equilibrium, as shown in the next section.

\section{Equilibrium of Distributed Power Control in MulTiChanNel SYSTEMS}

In this section, we first consider the equilibrium characteristics of general multichannel power control schemes and then give a sufficient condition that will assure the uniqueness of the equilibrium for the energy-efficient power optimization.

We consider a general non-cooperative power control over multiple subchannels where each user selfishly chooses power allocation to maximize its own utility in an interference-limited environment. The utility, denoted by $U_{n}\left(\mathbf{p}_{n}, \mathbf{I}_{n}\left(\mathbf{p}_{-n}\right)\right)$, is assumed to be quasiconcave in $\mathbf{p}_{n}$ given $\mathbf{I}_{n}$, interference on all subchannels. $\mathbf{I}_{n}$ is a function of $\mathbf{p}_{-n}$ and is determined by (1). An example of utility,
$U_{n}\left(\mathbf{p}_{n}, \mathbf{I}_{n}\left(\mathbf{p}_{-n}\right)\right)$, is energy efficiency, $u_{n}$ in (6). The best response of the power allocation of User $n$ is denoted to be

$$
\begin{aligned}
\mathbf{p}_{n}^{o} & =F_{n}\left(\mathbf{p}_{-n}\right)=\tilde{F}_{n}\left(\mathbf{I}_{n}\left(\mathbf{p}_{-n}\right)\right) \\
& =\arg \max _{\mathbf{p}_{n}} U_{n}\left(\mathbf{p}_{n}, \mathbf{I}_{n}\left(\mathbf{p}_{-n}\right)\right) .
\end{aligned}
$$

The non-cooperative energy-efficient power optimization in (9) is an example of (15).

Since $U_{n}\left(\mathbf{p}_{n}, \mathbf{I}_{n}\left(\mathbf{p}_{-n}\right)\right)$ is continuous and quasi-concave in $\mathbf{p}_{n}$, the equilibrium always exists [15]. Denote the Jacobian matrix of $\tilde{F}_{n}$ at $\mathbf{I}_{n}$ to be $\frac{\partial \tilde{F}_{n}}{\partial \mathbf{I}_{n}}$ and the Jacobian matrix of $\mathbf{I}_{n}$ at $\mathbf{p}_{-n}$ to be $\frac{\partial \mathbf{I}_{n}}{\partial \mathbf{p}_{-n}}$. Denote $\|A\|$ to be the Frobenius norm of matrix $A=\left(a_{i j}\right)$, i.e. $\|A\|=\sqrt{\sum_{i, j} a_{i j}^{2}}$. We know that when a contraction mapping has a fixed point, the fixed point is unique [16]. Readily, we have the following sufficient condition, which comes from [15], which assures a unique equilibrium.

Theorem 3 (Uniqueness). In frequency selective channels, if for any User $n,\left\|F_{n}\left(\mathbf{p}_{-n}\right)-F_{n}\left(\check{\mathbf{p}}_{-n}\right)\right\|<\left\|\mathbf{p}_{-n}-\check{\mathbf{p}}_{-n}\right\|$ for any different $\mathbf{p}_{-n}$ and $\check{\mathbf{p}}_{-n}$, there exists one and only one equilibrium $\mathbf{p}^{*}$ in the non-cooperative power control game defined by (15).

Intuitively, Theorem 3 indicates that if other users change their transmission powers by some amount, the best power allocation of the user is altered by a lesser amount, then the equilibrium is unique. Note that the transmission powers of other users and the best response $F_{n}\left(\mathbf{p}_{-n}\right)$ in (15) are related through interference channel gains, which therefore determines the variation of the best response and whether the sufficient condition can be guaranteed. Stronger interference channel gains result in higher correlation and vice versa. The above two-user network illustrates an example where one subchannel has extremely strong interference channel gains. In this case, the sufficient condition is violated and there are multiple equilibria.

Based on Theorem 3, Theorem 4 explicitly shows the impact of interference channel gains on the number of equilibria and is proved in Appendix E.

Theorem 4 (Uniqueness). In frequency selective channels, if for any User $n$,

$$
\left\|\frac{\partial \mathbf{I}_{n}}{\partial \mathbf{p}_{-n}}\right\|<\frac{1}{\sup _{\mathbf{I}_{n}}\left\|\frac{\partial \tilde{F}_{n}}{\partial \mathbf{I}_{n}}\right\|},
$$

where $\sup _{\mathbf{I}_{n}}$ is the supremum on all feasible $\mathbf{I}_{n}$, there exists one and only one equilibrium $\mathbf{p}^{*}$ in the non-cooperative power control game defined by (15).

The Jacobian matrix of $F_{n}$ at $\mathbf{I}_{n}$ is

$$
\frac{\partial \tilde{F}_{n}}{\partial \mathbf{I}_{n}}=\left(\begin{array}{ccc}
\frac{\partial p_{n}^{(1) o}}{\partial I_{n}^{(1)}} & \cdots & \frac{\partial p_{n}^{(K) o}}{\partial I_{n}^{(1)}} \\
\vdots & \ddots & \vdots \\
\frac{\partial p_{n}^{(1) o}}{\partial I_{n}^{(K)}} & \cdots & \frac{\partial p_{n}^{(K) o}}{\partial I_{n}^{(K)}}
\end{array}\right),
$$


while the Jacobian matrix of $\mathbf{I}_{n}$ at $\mathbf{p}_{-n}$ is

$$
\frac{\partial \mathbf{I}_{n}}{\partial \mathbf{p}_{-n}}=\left(\begin{array}{ccc}
g_{1 n}^{(1)} & & \mathbf{0} \\
& \ddots & \\
\mathbf{0} & & g_{1 n}^{(K)} \\
g_{(n-1) n}^{(1)} & \vdots & \\
g_{(n+1) n}^{(1)} & & \mathbf{0} \\
\mathbf{0} & \ddots & g_{(n-1) n}^{(K)} \\
& \vdots & \\
g_{N n}^{(1)} & & \mathbf{0} \\
\mathbf{0} & \ddots & \\
& & g_{N n}^{(K)}
\end{array}\right),
$$

where $\mathbf{0}$ indicates all elements at that side are 0 . Hence, the left hand side of (16) is

$$
\left\|\frac{\partial \mathbf{I}_{n}}{\partial \mathbf{p}_{-n}}\right\|=\sqrt{\sum_{i=1, i \neq n}^{N} \sum_{k=1}^{K} g_{i n}^{(k) 2}},
$$

which measures the strength of the interference on all subchannels that all other users bring to User $n$. It is easy to see that $\left\|\frac{\partial \mathbf{I}_{n}}{\partial \mathbf{p}_{-n}}\right\|$ depends only on the interference channel gains, which is mostly determined by the relative locations of different communication pairs that interfere with each other, i.e. how closely the network is coupled. On the other hand, the right hand side of (16) is independent of interference channel gains as it is the supreme of $\left\|\frac{\partial \tilde{F}_{n}}{\partial \mathbf{I}_{n}}\right\|$ on all possible interference channels. Furthermore, $\sup _{\mathbf{I}_{n}}\left\|\frac{\partial \tilde{F}_{n}}{\partial \mathbf{I}_{n}}\right\|$ depends on signal channel gains. Hence, the number of equilibria is determined by how strong interference channel gains and signal channel gains are. Consider an example where different users are sufficiently far away and all interference channel gains are close to zero. It is easy to see that transmission powers of other users have almost no effect on the best response of the user and there is a unique equilibrium. However, when users are moving closer to each other, (19) is getting larger and larger and once they are close enough such that (16) is violated, there may be multiple equilibria.

Note that while a sufficient condition of a unique equilibrium for distributed power control over a single channel is given in [14], we provide sufficient conditions of a unique equilibrium for distributed multichannel power controls in Theorems 3 and 4, which can be applied to different kinds of distributed multiple input multiple output (MIMO) and orthogonal frequency-division multiplexing (OFDM) systems.

Given Theorems 3 and 4, a sufficient condition to assure a unique equilibrium of the non-cooperative energy-efficient power optimization follows immediately.

Theorem 5 (Uniqueness). In frequency selective channels, the non-cooperative energy-efficient power optimization game defined by (9) has a unique equilibrium if for any User $n$, $\left\|f_{n}\left(\mathbf{p}_{-n}\right)-f_{n}\left(\check{\mathbf{p}}_{-n}\right)\right\|<\left\|\mathbf{p}_{-n}-\check{\mathbf{p}}_{-n}\right\|$ for any different $\mathbf{p}_{-n}$ and $\check{\mathbf{p}}_{-n}$ or $\left\|\frac{\partial \mathbf{I}_{n}}{\partial \mathbf{p}_{-n}}\right\|<\frac{1}{\sup _{\mathbf{I}_{n}}\left\|\frac{\partial f_{n}}{\partial \mathbf{I}_{n}}\right\|}$.

Note that the above theorem only gives sufficient conditions of uniqueness that may not be necessary ones. For example for a single-channel network, due to the strict concavity of $f_{n}\left(\mathbf{p}_{-n}\right), \sup _{I_{n}}|| \frac{\partial f_{n}}{\partial I_{n}} \|=\left.\frac{\partial f_{n}}{\partial I_{n}}\right|_{I_{n}=0}$. However, for all interference channel gains, there is always a unique equilibrium, as shown in Theorem 2.

\section{Tradeoff between Spectral and Energy EFFICIENCY}

In this section, we investigate the tradeoff between noncooperative energy-efficient and spectral-efficient power control schemes. To facilitate analysis and get insights, consider a symmetric single-channel network. There are $N$ users, all experiencing the same channel power gain $g$. All interference channels have the same power gain $\widetilde{g}$. To characterize interference level, we need to use a metric that is independent of transmission powers. Define the network coupling factor

$$
\alpha=\frac{\widetilde{g}}{g},
$$

which characterizes what level different links interfere with each other. Higher $\alpha$ represents a heavier interfering scenario. According to Theorem 2, the equilibrium state of noncooperative energy-efficient power optimization is unique. Due to the assumption of network symmetry, all users transmit with the same power in the equilibrium. Denote the transmission power of all users to be $p$.

The overall network EE will be

$$
\begin{aligned}
u(p) & =\sum_{n=1}^{N} \frac{w \log \left(1+\frac{p g}{\sum_{i, i \neq n} p \widetilde{g}+\sigma^{2}}\right)}{p+p_{c}} \\
& =\frac{N w \log \left(1+\frac{p}{(N-1) \alpha p+\frac{\sigma^{2}}{g}}\right)}{p+p_{c}},
\end{aligned}
$$

and the network SE will be

$$
r(p)=N \log \left(1+\frac{p}{(N-1) \alpha p+\frac{\sigma^{2}}{g}}\right) .
$$

With non-cooperative spectral-efficient power control, every user allocates power to selfishly maximize its SE. Without power limit, the transmission power tends to infinity in the equilibrium. Besides, we can see that $r(p)$ is strictly increasing in $p$. Hence, the maximum network $\mathrm{SE}$ is obtained in the equilibrium and the upperbound is

$$
r_{S E}=\lim _{p \rightarrow \infty} r(p)=N \log \left(1+\frac{1}{(N-1) \alpha}\right)
$$

with the corresponding EE $u_{S E}=\lim _{p \rightarrow \infty} u(p)=0$, which is completely energy inefficient and non-cooperative SE optimal power control is not desired for energy efficiency.

With non-cooperative energy-efficient power optimization, the network energy efficiency at the equilibrium is $u_{E E}=$ 


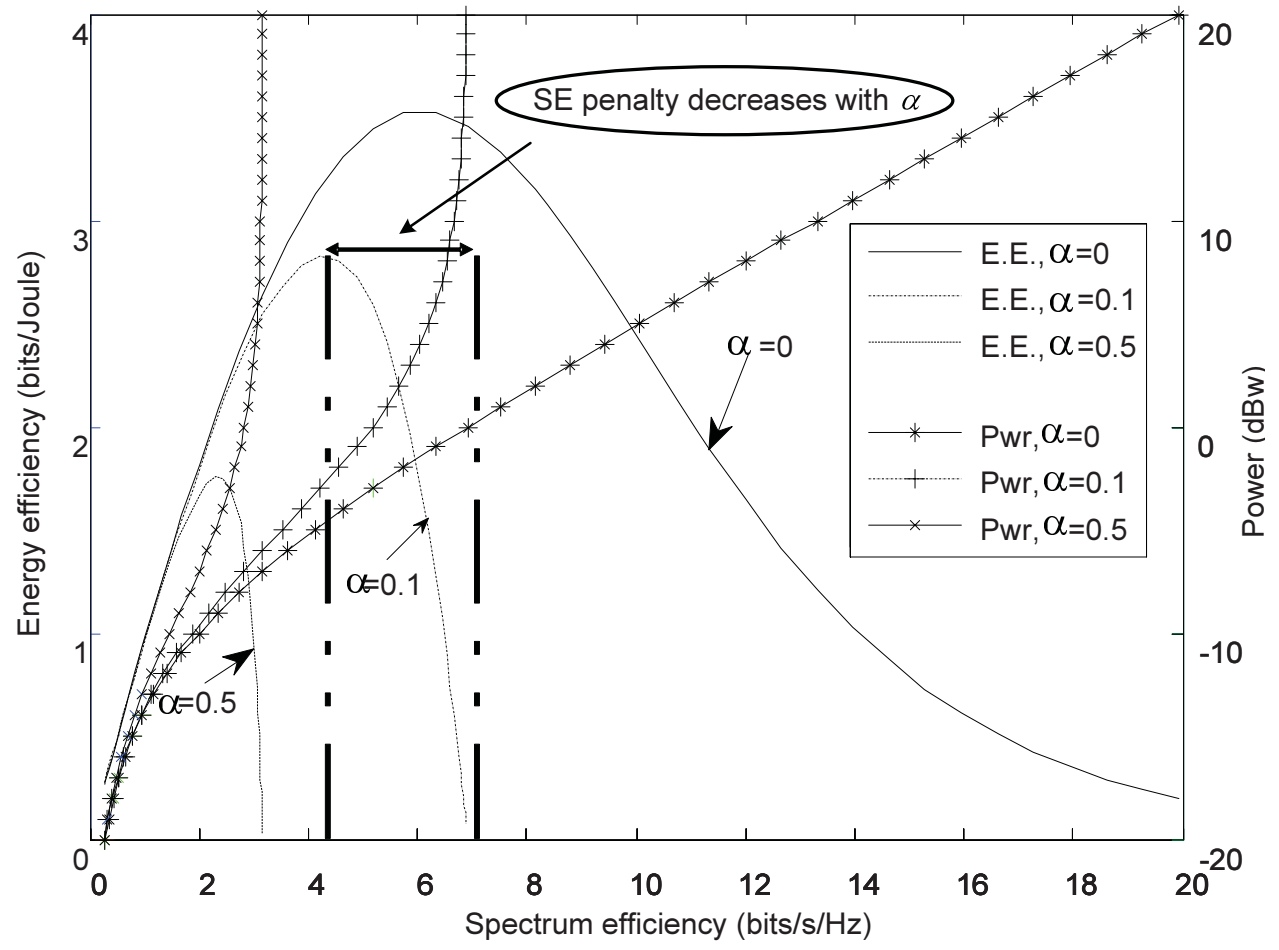

Fig. 1. Tradeoff of EE and SE with different interfering scenarios: left $\mathrm{Y}$ axis is for curves without markers and indicates the achieved EE while the SE is as in $\mathrm{X}$ axis; right $\mathrm{Y}$ axis is for curves with markers and indicates the required transmission power to achieve the spectral efficiency given in $\mathrm{X}$ axis. $\left(p_{c}=1, g=1, \sigma^{2}=0.01, N=2\right)$.

$u\left(p^{*}\right)$ with the corresponding SE $r_{E E}=r\left(p^{*}\right)$. Hence, the SE penalty of energy-efficient power optimization is

$$
r_{t r}=r_{S E}-r_{E E}=N \log \left(1+\frac{1}{(N-1) \alpha}\right)-r\left(p^{*}\right)
$$

In an interference-free scenario, i.e. $N=1$ or $\alpha=0$, the penalty is infinite. Otherwise, whenever interference exists, it is bounded.

To further understand the tradeoff, Figure 1 illustrates a case when two users transmit with the same power and interfere with each other. Curves with markers draw the relationship between transmission power and SE when the network has different couplings while those without markers draw the corresponding energy efficiency. When $\alpha=0$, arbitrary SE can be obtained by choosing enough transmission power. When $\alpha>0$, regions beyond the SE upperbound is not achievable. Furthermore, EE is much more sensitive to power selection than SE. For example when $\alpha=0.1$, the transmission power is chosen to be $-3 \mathrm{dBw}$ for energy-efficient power optimization. The SE achieved is $4.2 \mathrm{bits} / \mathrm{s} / \mathrm{Hz}$ while the $\mathrm{EE}$ is $2.8 \mathrm{bits} / \mathrm{Joule}$. If we further increase the transmission power, the EE decreases very fast while the SE only improves slightly. Hence, in interference-limited scenarios, increasing transmission power beyond the optimal power for EE has little SE improvement but significantly hurts EE. Furthermore, power optimization to achieve the highest energy efficiency will also have reduced SE penalty with the increase of $\alpha$. Figure 2 shows the transmission power in the equilibrium when the network has different couplings and numbers of users. The equilibrium power decreases with either user number or $\alpha$ and automatically alleviates network interference.

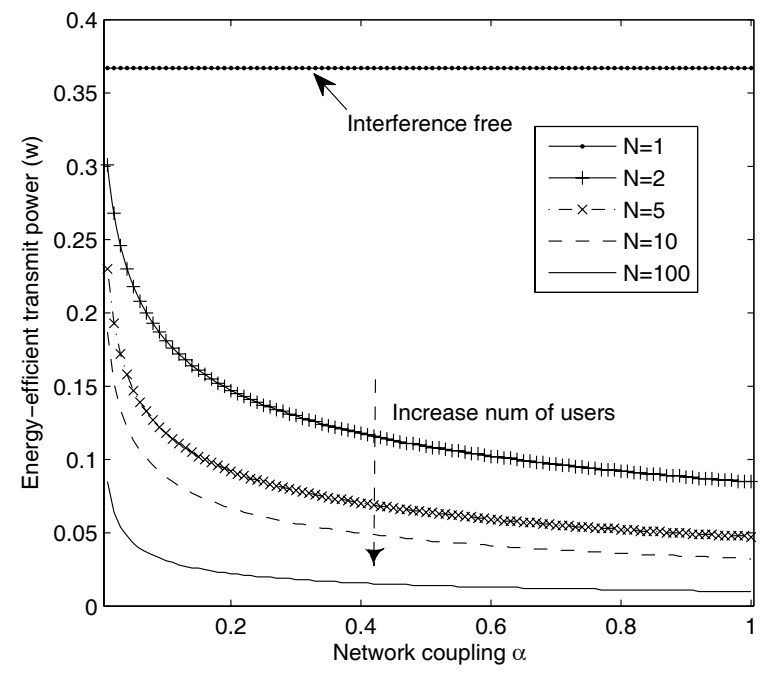

Fig. 2. Non-cooperative energy-efficient power optimization in the equilibrium $\left(P_{c}=1, g=1, \sigma^{2}=0.01\right)$.

\section{IMPLEMENTATION ISSUES}

In the previous section we know that energy-efficient power optimization is advantageous in interference-limited scenarios due to its conservative power allocation nature. In this section, we will develop practical approaches for non-cooperative energy-efficient power optimization.

In (9), the best response of User $n$ depends on the transmission power vectors of all other users, $\mathbf{p}_{-n}$, which can not be obtained in a non-cooperative setting. Instead, we observe that $\mathbf{p}_{-n}$ affects the best response in the form of interference, which thus contains sufficient information of $\mathbf{p}_{-n}$ to determine 
the best response and can be acquired locally. Hence, we let each user measure interferences on all subchannels to determine the power optimization.

At time $t-1$, the measured interference powers on all subchannels of User $n$ are denoted by $\mathbf{I}_{n}[t-1]=\left[I_{n}^{(1)}[t-\right.$ 1], $\left.I_{n}^{(2)}[t-1], \cdots, I_{n}^{(K)}[t-1]\right]$. Denote the predicted SINR to be

$$
\widehat{\eta}_{n}^{(k)}[t]=\frac{p_{n}^{(k)}[t] g_{n n}^{(k)}}{\widehat{I}_{n}^{(k)}[t]+\sigma^{2}}=\frac{p_{n}^{(k)}[t] g_{n n}^{(k)}}{I_{n}^{(k)}[t-1]+\sigma^{2}} .
$$

Hence, the predicted EE is

$$
\widehat{u}_{n[t]}\left(\mathbf{p}_{n}[t]\right)=\frac{\widehat{r}_{n}[t]}{p_{n}[t]+p_{c}}=\frac{\sum_{k} R\left(\widehat{\eta}_{n}^{(k)}[t]\right)}{\sum_{k} p_{n}^{(k)}[t]+p_{c}} .
$$

The best response at time $t$ of User $n$ is

$$
\mathbf{p}_{n}^{o}[t]=\underset{\mathbf{p}_{n}[t]}{\arg \max } \widehat{u}_{n[t]}\left(\mathbf{p}_{n}[t]\right) .
$$

Due to the strict quasi-concavity of $\widehat{u}_{n[t]}$, numerical methods like gradient ascent algorithms can be used to find the optimal power allocation at each time slot. A Binary Search Assisted Ascent algorithm has been developed in [9]. However, if we obtain the optimal power allocation at each time slot, it requires intensive computations. Instead, we introduce a temporal iterative binary search (TIBS) algorithm to track channel temporal variation and search for the optimal power allocation with reduced complexity.

The basic idea of TIBS is to search a better power allocation along the gradient at each time slot and enable iterative search along time. The power at $t$ is updated by

$$
\mathbf{p}_{n}[t]=\mathbf{p}_{n}[t-1]+\mu\left(\nabla \widehat{u}_{n[t]}\right)_{\mathbf{p}_{n}[t-1]},
$$

where $\left(\nabla \widehat{u}_{n[t]}\right)_{\mathbf{p}_{n}[t-1]}$ is the gradient of $\widehat{u}_{n[t]}$ at $\mathbf{p}_{n}[t-1]$ and $\mu$ is a small step size. Fixing channel states and transmission powers of all other users, the EE at $t$ will always be bigger than that at $t-1$ with sufficiently small step size except when the gradient is zero, i.e. $\mathbf{p}_{n}[t-1]$ is already optimal [17]. However, small step size leads to slow convergence and channel tracking capability. Denote

$$
g(\mu)=\widehat{u}_{n[t]}\left(\mathbf{p}_{n}[t-1]+\mu\left(\nabla \widehat{u}_{n[t]}\right)_{\mathbf{p}_{n}[t-1]}\right) .
$$

It is easy to show that $g(\mu)$ is also strictly quasi-concave in $\mu$ and binary search can be used for rapid location of the optimal step size $\mu^{*}$ to find $\mathbf{p}_{n}^{o}[t]$ [9]. The TIBS algorithm is summarized in the following.

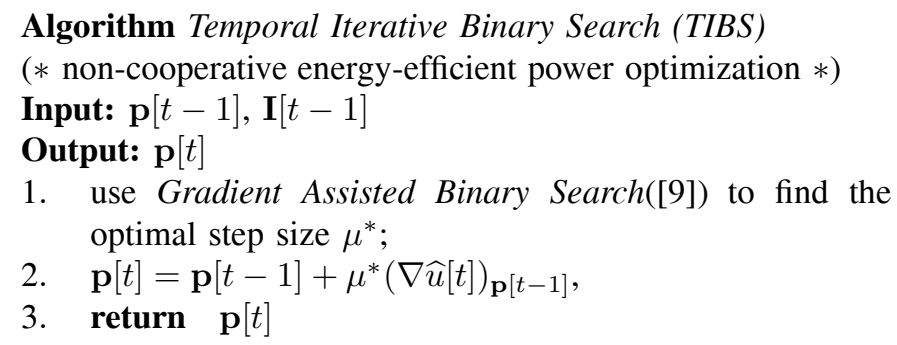

\section{Simulation Results}

In this section, we present simulation results for an interference-limited uplink OFDMA cellular network and observe the performance of the network in equilibrium.
TABLE I

SYSTEM PARAMETERS

\begin{tabular}{|c|c|}
\hline Carrier frequency & $1.5 \mathrm{GHz}$ \\
\hline Number of subchannels & 96 \\
\hline Subchannel bandwidth & $10 \mathrm{kHz}$ \\
\hline Target BER & $10^{-3}$ \\
\hline Thermal noise power, $N_{o}$ & $-141 \mathrm{dBW} / \mathrm{MHz}$ \\
\hline Circuit power, $P_{C}$ & $100 \mathrm{~mW}$ \\
\hline Maximum transmission power & $33 \mathrm{dBm}$ \\
\hline Propagation model & Okumura-Hata model \\
\hline Shadowing & Log-normal \\
\hline Fading & Rayleigh flat fading \\
\hline Modulation & Uncoded M-QAM \\
\hline
\end{tabular}

The network consists of seven hexagonal cells and the center cell is surrounded by the other six. The frequency reuse factor is one. Users are uniformly dropped into each cell at each simulation trial. The system parameters are listed in Table I. The base station schedules subchannels to maximize different network performance metrics. All schedulers and corresponding power control schemes are listed in Table II. Since the energy-efficient scheduling for frequency-selective channels is still an open problem, we use Rayleigh flatfading channel and apply the energy-efficient schedulers both with and without proportional fairness for flat-fading OFDMA in [7]. The traditional proportional fair scheduler assigns subchannels to the user with the highest $\frac{r}{T}$, where $r$ is the instantaneous data rate on that subchannel and $T$ the average total throughput [18]. While energy-efficient schedulers assign subchannels to different users to maximize EE either with or without fairness, the traditional proportional fair scheduler assigns all subchannels to one user at each time slot due to flat fading. We also implement a soft power control scheme proposed in $802.16 \mathrm{~m} \mathrm{[19].} \mathrm{In} \mathrm{this} \mathrm{scheme,} \mathrm{transmission} \mathrm{power}$ is controlled based on interference strength and path loss. The parameters in the soft power control scheme are selected to maximize the throughput of cell-edge users while not hurting the throughput of other users too much.

Figure 3 compares the average sum network EE and the corresponding throughput performance respectively. For fixedpower transmission, the transmission powers are shown in the legend. To see performance loss due to interference, the energy-efficient scheduler without fairness and the traditional proportional scheduler with the maximum transmission power is also simulated in a single cell network. We can see that transmitting with the highest power brings the highest interference and causes significant throughput loss for the traditional scheduler. In contrast, energy-efficient power control effectively reduces network interference and has much less throughput loss. While our previous results in [7] show that $\mathrm{EE}$ and throughput efficiency do not necessarily agree for an interference-free single cell scenario, the situation is different for a multi-cell interference-limited network. Here energy-efficient schemes optimize both throughput and energy utilization and exhibit an improved SE tradeoff.

Figure 4 further shows the cumulative distribution functions (CDFs) of energy efficiency and throughput when there are nine users in the network. Observe the throughput CDF of the soft power control scheme. Compared with other traditional schemes, it maximizes cell-edge throughput that is illustrated 


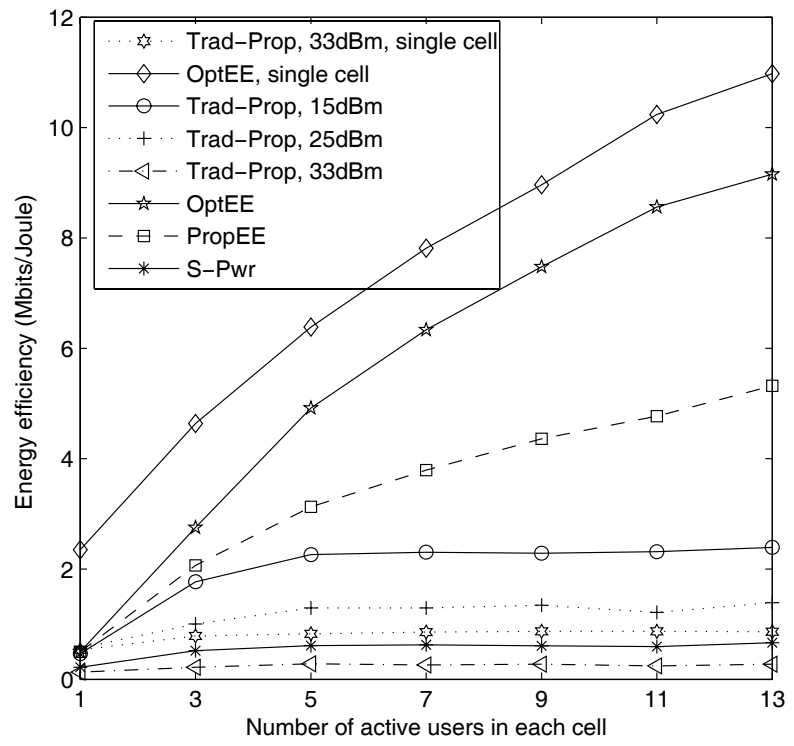

(a) Energy efficiency.

Fig. 3. Performance comparison of different schemes.

TABLE II

SCHEdUling AND POWER CONTROL

\begin{tabular}{|c|c|c|}
\hline Legend & Scheduler & Power control \\
\hline OptEE & $\begin{array}{c}\text { Energy-efficient } \\
\text { scheduler w/o fairness }\end{array}$ & TIBS \\
\hline PropEE & $\begin{array}{c}\text { Energy-efficient scheduler } \\
\text { w/ proportional fairness }\end{array}$ & TIBS \\
\hline Trad-Prop & Traditional proportional fair & Fixed power \\
\hline S-Pwr & Traditional proportional fair & Adaptive power control \\
\hline
\end{tabular}

in low-throughput range. However, it performs much worse than other traditional schemes in high-throughput range. From the CDFs, we can see that the proposed EE schemes not only improve the sum energy efficiency and throughput, but also uniformly improve the performance of all users in the cell.

\section{CONCLUSION}

We investigated energy-efficient power optimization schemes for interference-limited communications in this paper. We developed a non-cooperative energy-efficient power optimization game. We further studied the properties of the equilibrium for this game and showed that the equilibrium of this game always exists. When there is only one subchannel or the channel experiences flat fading, there will be a unique equilibrium. However, in frequency-selective channels, this is not true in general. We have found a sufficient condition that assures the uniqueness and developed a practical approach of the non-cooperative power optimization game. Our study on the existence and uniqueness of equilibrium guarantees that the system is stable using non-cooperative energy efficient power optimization. Since theoretical equilibrium analysis of the network is difficult because of the non-convexity of the energy efficiency function as well as the multi-channel and multi-user dimension of the power control, we use Monte Carlo simulation to demonstrate that, the proposed scheme improves not only energy efficiency but also spectral efficiency uniformly for all users due to the conservative nature of power optimization, which reduces other-cell

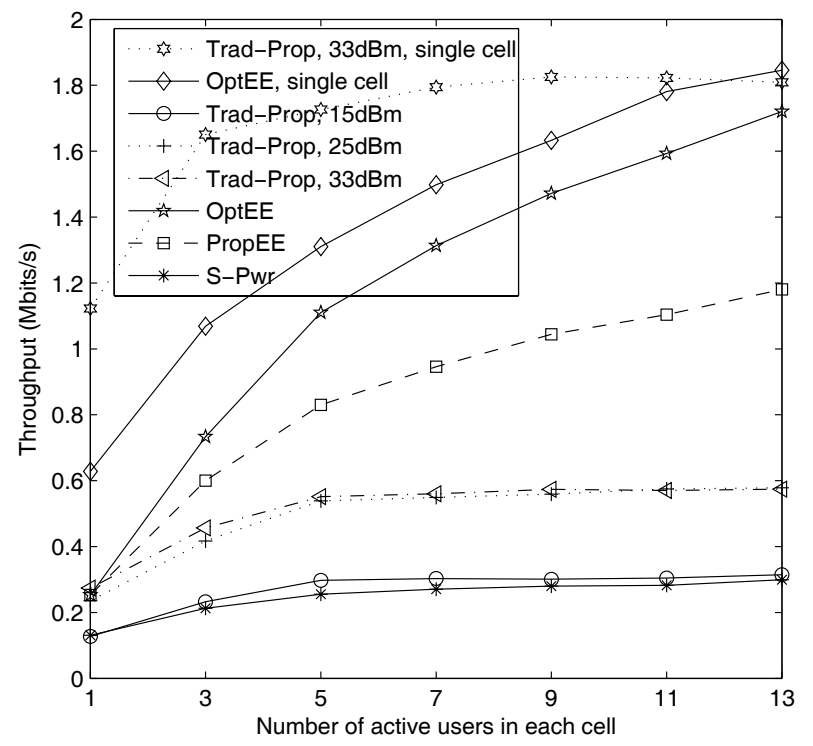

(b) Throughput.

interference to improve the overall network throughput. The proposed scheme can be applied in different types of networks, e.g. cellular networks, ad hoc networks and sensor networks, to improve system spectral efficiency and device energy efficiency.

\section{ACKNOWLEDGMENT}

The authors would like to thank Ali T. Koc, Dave Bormann, Changho Suh, Hongseok Kim, and Ozgur Oyman for helpful discussions.

\section{APPENDIX A \\ PROOF OF LEMMA 1}

Proof: Denote the upper contour sets of $u_{n}\left(\mathbf{p}_{n}, \mathbf{p}_{-n}\right)$ as $S_{\alpha}=\left\{\mathbf{p}_{n} \succeq \mathbf{0} \mid u_{n}\left(\mathbf{p}_{n}, \mathbf{p}_{-n}\right) \geq \alpha\right\}$, where symbol $\succeq$ denotes vector inequality and $\mathbf{R} \succeq \mathbf{0}$ means each element of $\mathbf{R}$ is non-negative. According to Proposition C.9 of [13], $u_{n}\left(\mathbf{p}_{n}, \mathbf{p}_{-n}\right)$ is strictly quasiconcave in $\mathbf{p}_{n}$ if and only if $S_{\alpha}$ is strictly convex for any real number $\alpha$. It is obvious that when $\alpha \leq 0, S_{\alpha}$ is strictly convex. Now we investigate the case when $\alpha>0$. Since $u_{n}\left(\mathbf{p}_{n}, \mathbf{p}_{-n}\right)=$ $\frac{\sum_{k=1}^{K} R\left(\frac{p_{n}^{(k)} g_{n n}^{(k)}}{\sum_{i=1, i \neq n}^{N} p_{i}^{(k)} g_{i n}^{(k)}+\sigma^{2}}\right)}{p_{c}+\sum_{k=1}^{K} p_{n}^{(k)}} \geq \alpha, \quad S_{\alpha}$ is equivalent to $S_{\alpha}=\left\{\mathbf{p}_{n} \succeq \mathbf{0} \mid \sum_{k=1}^{K} R\left(\frac{p_{n}^{(k)} g_{n n}^{(k)}}{\sum_{i=1, i \neq n}^{N} p_{i}^{(k)} g_{i n}^{(k)}+\sigma^{2}}\right)-\left(p_{c}+\right.\right.$ $\left.\left.\sum_{k=1}^{K} p_{n}^{(k)}\right) \alpha \geq 0\right\}$. Since $R()$ is strictly concave in SINR, $R()$ of User $n$ is also strictly concave in the transmission power $p_{n}^{(k)}$ given the transmission powers of all other users, i.e. $p_{i}^{(k)}$ where $i \neq n$. Obviously, $S_{\alpha}$ is also strictly convex. Hence, we have Lemma 1.

\section{APPENDIX B \\ PROOF OF THEOREM 1}

Proof: In [15], it has been shown a Nash equilibrium exists in a non-cooperative game if for any $n,(1) \mathbf{p}_{n}$ is a nonempty, convex, and compact subset of some Euclidean space 


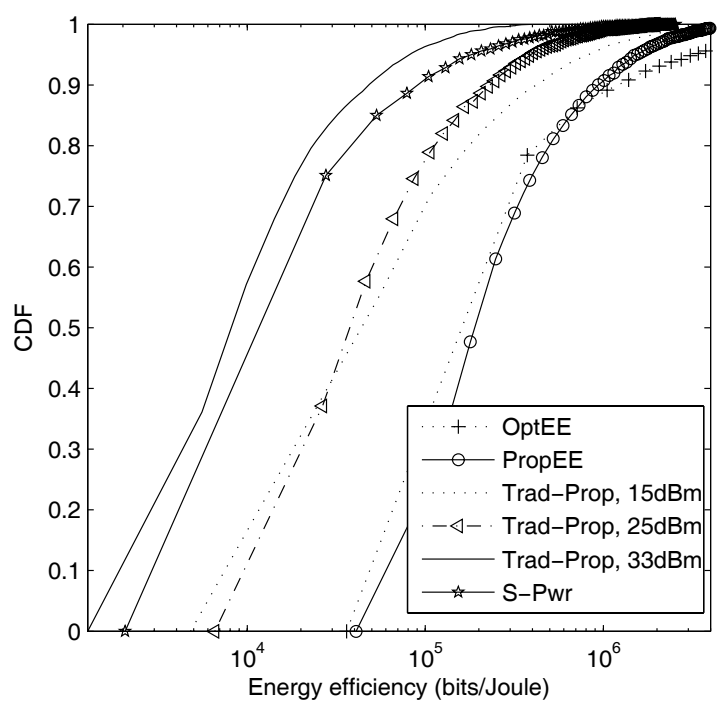

(a) Energy efficiency.

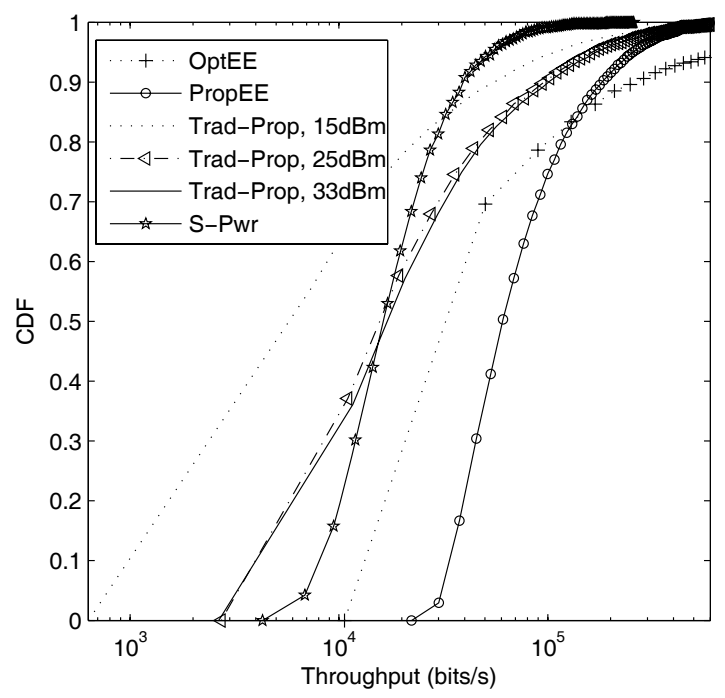

(b) Throughput.

Fig. 4. Comparison of different schemes.

$\mathfrak{R}^{L}$ and (2) $u_{n}\left(\mathbf{p}_{n}, \mathbf{p}_{-n}\right)$ is continuous and quasi-concave in $\mathbf{p}_{n}$, both of which are satisfied in our non-cooperative energyefficient control game. Hence, the existence of the equilibrium immediately follows. According to our previous work in [9], in a point-to-point energy-efficient transmission, the necessary and sufficient condition for a data rate vector of User $n, \mathbf{r}_{n}^{o}=$ $\left[r_{n}^{(1) o}, r_{n}^{(2) o}, \cdots, r_{n}^{(K) o}\right]$, to be globally optimal is given by, for any subchannel $i$,

(i) if

$$
\begin{aligned}
& \text { if } \frac{p_{c}+\sum_{j \neq i} p_{n}^{(j)}}{\sum_{j \neq i} r_{n}^{(j)}} \geq\left.\quad \frac{\partial\left(\sum_{j} p_{n}^{(j)}\right)}{\partial r_{n}^{(i)}}\right|_{\mathbf{r}_{n}=\mathbf{r}_{n}^{(i 0)}}, \\
& \left.\frac{\partial u_{n}\left(\mathbf{p}_{n}, \mathbf{p}_{-n}\right)}{\partial r_{n}^{(i)}}\right|_{\mathbf{r}_{n}=\mathbf{r}_{n}^{o}}=0 \text {, i.e. }\left.\frac{\partial\left(\sum_{j} p_{n}^{(j)}\right)}{\partial r_{n}^{(i)}}\right|_{\mathbf{r}_{n}=\mathbf{r}_{n}^{o}}= \\
& \frac{1}{u\left(\mathbf{p}_{n}^{o}, \mathbf{p}_{-n}\right)} ;
\end{aligned}
$$

(ii) otherwise, $r_{n}^{(i) o}=0$,

where

$$
\mathbf{r}_{n}^{(i 0)}=\left[r_{n}^{(1) o}, r_{n}^{(2) o}, \cdots, r_{n}^{(i-1) o}, 0, r_{n}^{(i+1) o}, \cdots, r_{n}^{(K) o}\right] .
$$

By transformation of parameters, $\frac{\partial f}{\partial r_{n}^{(i)}}=\frac{\partial f}{\partial p_{n}^{(i)}} / \frac{\partial r_{n}^{(i)}}{\partial p_{n}^{(i)}}=$ $\frac{\partial f}{\partial p_{n}^{(i)}} \frac{1}{R^{\prime}\left(\eta_{n}^{(i)}\right) \gamma_{n}^{(i)}}$, where $R^{\prime}()$ is the first order derivative of $R()$ and $\gamma_{n}^{(i)}=\frac{\eta_{n}^{(i)}}{p_{n}^{(i)}}=\frac{g_{n n}^{(i)}}{\sum_{j=1, j \neq n}^{N} p_{j}^{(i)} g_{j n}^{(i)}+\sigma^{2}}$. Hence, we have the following equivalent condition for each user. For any subchannel $i$, (i) if $\frac{\sum_{j \neq i} r_{n}^{(j)}}{p_{c}+\sum_{j \neq i} p_{n}^{(j)}} \leq R^{\prime}(0) \gamma_{n}^{(i)},\left.\frac{\partial u_{n}\left(\mathbf{p}_{n}, \mathbf{p}_{-n}\right)}{\partial p_{n}^{(i)}}\right|_{\mathbf{p}_{n}=\mathbf{p}_{n}^{o}}=0$,
i.e.

$$
R^{\prime}\left(\gamma_{n}^{(i)} p_{n}^{(i) o}\right) \gamma_{n}^{(i)}=u\left(\mathbf{p}_{n}^{o}, \mathbf{p}_{-n}\right) ;
$$

(ii) otherwise, $p_{n}^{(i) o}=0$.

It is easy to see that the network achieves an equilibrium if and only if the power settings of all users satisfy the above conditions. Theorem 1 is readily obtained. $f_{n}\left(\mathbf{p}_{-n}\right)>0$ and we have the positivity. Denote $I_{n}=$ $\sum_{j=1, j \neq n}^{N} p_{j} g_{j n}$ and $\gamma_{n}=\frac{g_{n n}}{I+\sigma^{2}}$. According to (B.30), $p_{n}^{o}$ satisfies

$$
R^{\prime}\left(\gamma_{n} p_{n}^{o}\right) \gamma_{n}=u\left(p_{n}^{o}, \mathbf{p}_{-n}\right)=\frac{R\left(\gamma_{n} p_{n}^{o}\right)}{p_{c}+p_{n}^{o}} .
$$

Substituting $R(\eta)=w \log (1+\eta)$ in to (C.31), we have the following equivalent condition,

$$
\begin{aligned}
& Q\left(p_{n}^{o}, I\right)= \\
& g_{n n}\left(p_{c}+p_{n}^{o}\right)-\left(p_{n}^{o} g_{n n}+I+\sigma^{2}\right) \log \left(1+\frac{p_{n}^{o} g_{n n}}{I+\sigma^{2}}\right) \\
& =0 .
\end{aligned}
$$

Hence, $\frac{\partial p_{n}^{o}}{\partial I}=-\frac{\partial Q}{\partial I} / \frac{\partial Q}{\partial p_{n}^{o}}=\frac{p_{n}^{o} \gamma_{n}-\log \left(1+p_{n}^{o} \gamma_{n}\right)}{g_{n n} \log \left(1+p_{n}^{o} \gamma_{n}\right)}$. Since $x>$ $\log (1+x)$ for all $x>0$, we have $\frac{\partial p_{n}^{o}}{\partial I}>0$. The monotonicity follows immediately. Furthermore,

$$
\begin{aligned}
\frac{\partial^{2} p_{n}^{o}}{\partial I^{2}} & =\frac{\partial \frac{\partial p_{n}^{o}}{\partial I}}{\partial I} \\
& =-\frac{p_{n}^{o}\left(-p_{n}^{o} \gamma_{n}+\left(1+p_{n}^{o} \gamma_{n}\right) \log \left(1+p_{n}^{o} \gamma_{n}\right)\right)}{\left(I+\sigma^{2}\right)\left(I+\sigma^{2}+p_{n}^{o} g_{n n}\right) \log \left(1+p_{n}^{o} \gamma_{n}\right)^{2}}
\end{aligned}
$$

We can easily show that $(1+x) \log (1+x)>x$ for all $x>0$ since $(1+0) \log (1+0)=0$ and $(1+x) \log (1+x)-x$ has positive first-order derivative when $x>0$. Thus, $\frac{\partial^{2} p_{n}^{o}}{\partial I^{2}}<0$. Since $I$ is a linear combination of $\mathbf{p}_{-n}, f_{n}\left(\mathbf{p}_{-n}\right)$ is strictly concave in $\mathbf{p}_{-n}$. Let $F(\alpha)=\alpha f_{n}\left(\mathbf{p}_{-n}\right)-f_{n}\left(\alpha \mathbf{p}_{-n}\right)$ and we need to show $F(\alpha)>0$ for all $\alpha>1$ to prove the scalability. Note that $F(1)=0$ and $\frac{\partial^{2} F(\alpha)}{\partial \alpha^{2}}>0$, it is sufficient to show that $\left.\frac{\partial F(\alpha)}{\partial \alpha}\right|_{\alpha=1}=f_{n}\left(\mathbf{p}_{-n}\right)-\mathbf{p}_{-n} f_{n}^{\prime}\left(\mathbf{p}_{-n}\right)>0$, which is obvious because of the positivity and concavity of $f_{n}\left(\mathbf{p}_{-n}\right)$.

\section{APPENDIX C}

\section{PROOF OF LEMMA 2}

Proof: $p_{n}^{o}=f_{n}\left(\mathbf{p}_{-n}\right)=\arg \max _{p_{n}} u_{n}\left(p_{n}, \mathbf{p}_{-n}\right)$. Since $u_{n}\left(0, \mathbf{p}_{-n}\right)=0$ and $u_{n}\left(p_{n}, \mathbf{p}_{-n}\right)>0$ for any $p_{n}>0$, 


\section{APPENDIX D \\ Proof of Two Equilibria In FREQUENCY-SELECTIVE CHANNELS}

Proof: We need to show that one of the equilibrium has the form $\mathbf{p}_{1}^{*}=\left[p_{\alpha} p_{\beta}\right]$ and $\mathbf{p}_{2}^{*}=\left[p_{\gamma} 0\right]$, where $p_{\alpha}, p_{\beta}$, and $p_{\gamma}$ are positive. We only need to verify that there exist $p_{\alpha}, p_{\beta}$, and $p_{\gamma}$ that satisfy Theorem 1 . Suppose $\mathbf{p}_{2}^{*}=\left[p_{\gamma} 0\right]$. After some calculation, it is easy to see that $\sigma^{2} \gg p_{\gamma} g_{21}^{(1)}$ and $\eta_{1}^{(1)} \approx \frac{p_{\alpha} g_{11}^{(1)}}{\sigma^{2}}$. Hence, both subchannels of User 1 have approximately the same SINR condition. Thus in the equilibrium, the transmission powers on the two subchannels of User 1 are almost the same. Besides, they cannot be zero. Hence, both are positive and satisfy the first condition of Theorem 1. Assume $\mathbf{p}_{1}^{*}=\left[p_{\alpha} p_{\beta}\right]$. Now we verify $\mathbf{p}_{2}^{*}$. Since User 2 does not transmit on the second subchannel, $\frac{\sum_{j \neq 1} r_{n}^{(j) *}}{p_{c}+\sum_{j \neq 1} p_{n}^{(j) *}}=0$ and the first condition of Theorem 1 should be satisfied. Hence, a positive power is allocated on the first subchannel in the equilibrium of User 2. Regarding the second subchannel, $\gamma_{n}^{(2) *}=\frac{g_{n n}^{(2)}}{p_{\beta} g_{12}^{(2)}+\sigma^{2}}=\frac{1}{p_{\beta} 1 e^{10}+1} \rightarrow 0$. Hence, $\frac{\sum_{j \neq 2} r_{2}^{(j) *}}{p_{c}+\sum_{j \neq 2} p_{n}^{(j) *}}>R^{\prime}(0) \gamma_{n}^{(2) *} \rightarrow 0$ and condition 2 of Theorem 1 is satisfied. Hence, $\mathbf{p}_{2}^{*}=\left[p_{\gamma} 0\right]$. Numerical methods can be used to determine the exact values of $p_{\alpha}, p_{\beta}$, and $p_{\gamma}$.

\section{APPENDIX E \\ PROOF OF THEOREM 4}

Proof: For any two power vectors $\mathbf{p}_{-n}$ and $\check{\mathbf{p}}_{-n}$, define the function $\mathfrak{F}_{n}(\theta)=F_{n}\left(\check{\mathbf{p}}_{-n}+\theta\left(\mathbf{p}_{-n}-\check{\mathbf{p}}_{-n}\right)\right)$. It is clear that $\mathfrak{F}_{n}(0)=F_{n}\left(\check{\mathbf{p}}_{-n}\right)$ and $\mathfrak{F}_{n}(1)=F_{n}\left(\mathbf{p}_{-n}\right)$; By the chain rule, we know that $\frac{\partial \widetilde{\mathcal{F}}_{n}}{\partial \theta}=\left(\mathbf{p}_{-n}-\check{\mathbf{p}}_{-n}\right) \frac{\partial F_{n}}{\partial\left(\check{\mathbf{p}}_{-n}+\theta\left(\mathbf{p}_{-n}-\check{\mathbf{p}}_{-n}\right)\right)}$. Hence, we have

$$
\begin{aligned}
& F_{n}\left(\mathbf{p}_{-n}\right)-F_{n}\left(\check{\mathbf{p}}_{-n}\right)=\mathfrak{F}_{n}(1)-\mathfrak{F}_{n}(0) \\
& =\int_{0}^{1} \mathfrak{F}_{n}^{\prime}(\theta) d \theta \\
& =\left(\mathbf{p}_{-n}-\check{\mathbf{p}}_{-n}\right) \int_{0}^{1} \frac{\partial F_{n}}{\partial\left(\check{\mathbf{p}}_{-n}+\theta\left(\mathbf{p}_{-n}-\check{\mathbf{p}}_{-n}\right)\right)} d \theta .
\end{aligned}
$$

Thus,

$$
\begin{aligned}
& \left\|F_{n}\left(\mathbf{p}_{-n}\right)-F_{n}\left(\check{\mathbf{p}}_{-n}\right)\right\| \\
& =\left\|\left(\mathbf{p}_{-n}-\check{\mathbf{p}}_{-n}\right) \int_{0}^{1} \frac{\partial F_{n}}{\partial\left(\check{\mathbf{p}}_{-n}+\theta\left(\mathbf{p}_{-n}-\check{\mathbf{p}}_{-n}\right)\right)} d \theta\right\| \\
& \leq\left\|\left(\mathbf{p}_{-n}-\check{\mathbf{p}}_{-n}\right)\right\|\left\|\int_{0}^{1} \frac{\partial F_{n}}{\partial\left(\check{\mathbf{p}}_{-n}+\theta\left(\mathbf{p}_{-n}-\check{\mathbf{p}}_{-n}\right)\right)} d \theta\right\| \\
& \leq\left\|\left(\mathbf{p}_{-n}-\check{\mathbf{p}}_{-n}\right)\right\| \int_{0}^{1}\left\|\frac{\partial F_{n}}{\partial\left(\check{\mathbf{p}}_{-n}+\theta\left(\mathbf{p}_{-n}-\check{\mathbf{p}}_{-n}\right)\right)}\right\| d \theta \\
& \leq\left\|\left(\mathbf{p}_{-n}-\check{\mathbf{p}}_{-n}\right)\right\| \int_{0}^{1}\left\|\sup _{\mathbf{p}_{-n}} \frac{\partial F_{n}}{\partial \mathbf{p}_{-n}}\right\| d \theta \\
& =\left\|\left(\mathbf{p}_{-n}-\check{\mathbf{p}}_{-n}\right)\right\|\left\|\sup _{\mathbf{p}_{-n}} \frac{\partial F_{n}}{\partial \mathbf{p}_{-n}}\right\| .
\end{aligned}
$$

Besides, according to the chain rule,

$$
\frac{\partial F_{n}}{\partial \mathbf{p}_{-n}}=\frac{\partial \mathbf{I}_{n}}{\partial \mathbf{p}_{-n}} \frac{\partial \tilde{F}_{n}}{\partial \mathbf{I}_{-n}}
$$

Hence, we have

$$
\begin{aligned}
\frac{\left\|F_{n}\left(\mathbf{p}_{-n}\right)-F_{n}\left(\check{\mathbf{p}}_{-n}\right)\right\|}{\left\|\mathbf{p}_{-n}-\check{\mathbf{p}}_{-n}\right\|} & \leq \sup _{\mathbf{p}_{-n}}\left\|\frac{\partial F_{n}}{\partial \mathbf{p}_{-n}}\right\| \\
& =\sup _{\mathbf{p}_{-n}}\left\|\frac{\partial \mathbf{I}_{n}}{\partial \mathbf{p}_{-n}} \frac{\partial \tilde{F}_{n}}{\partial \mathbf{I}_{-n}}\right\| \\
& \leq\left\|\frac{\partial \mathbf{I}_{n}}{\partial \mathbf{p}_{-n}}\right\| \sup _{\mathbf{I}_{-n}}\left\|\frac{\partial \tilde{F}_{n}}{\partial \mathbf{I}_{-n}}\right\| ;
\end{aligned}
$$

When $\left\|\frac{\partial \mathbf{I}_{n}}{\partial \mathbf{p}_{-n}}\right\|<\frac{1}{\sup _{\mathbf{I}_{n}}\left\|\frac{\partial \tilde{F}_{n}}{\partial \mathbf{I}_{n}}\right\|}, \frac{\left\|F_{n}\left(\mathbf{p}_{-n}\right)-F_{n}\left(\check{\mathbf{p}}_{-n}\right)\right\|}{\left\|\mathbf{p}_{-n}-\check{\mathbf{p}}_{-n}\right\|}<1$. The uniqueness of equilibrium follows immediately from Theorem 3.

\section{REFERENCES}

[1] Draft IEEE std 802.16e/D9, "IEEE standard for local and metropolitan area networks-part 16: air interface for fixed and mobile broadband wireless access systems," June 2005.

[2] D. Goodman and N. Mandayam, "Power control for wireless data," IEEE Personal Commun. Mag., vol. 7, no. 2, pp. 48-54, Apr. 2000.

[3] D. Gesbert, S. G. Kiani, A. Gjendemsj, and G. E. Ien , "Adaptation, coordination, and distributed resource allocation in interference-limited wireless networks," Proc. IEEE, vol. 95, no. 12, pp. 2393-2409, Dec. 2007.

[4] A. Gjendemsj, D. Gesbert, G. E. ien, and S. G. Kiani, "Optimal power allocation and scheduling for two-cell capacity maximization," in Proc. RAWNET, Apr. 2006.

[5] K. Lahiri, A. Raghunathan, S. Dey, and D. Panigrahi, "Battery-driven system design: a new frontier in low power design," in Proc. Intl. Conf. VLSI Design, Jan. 2002, pp. 261-267.

[6] F. Meshkati, H. V. Poor, and S. C. Schwartz, "Energy-efficient resource allocation in wireless networks," IEEE Commun. Mag., pp. 58-68, May 2007.

[7] G. W. Miao, N. Himayat, Y. Li, and D. Bormann, "Energy-efficient design in wireless OFDMA," in Proc. IEEE ICC, May 2008, pp. 33073312.

[8] G. Miao, N. Himayat, and Y. Li, "Energy-efficient transmission in frequency-selective channels," in Proc. IEEE Globecom, Nov. 2008, pp. $1-5$.

[9] _ , "Energy-efficient link adaptation in frequency-selective channels," IEEE Trans. Commun., vol. 58, no. 2, pp. 545-554, Feb. 2010.

[10] G. W. Miao, N. Himayat, G. Y. Li, and S. Talwar, "Low-complexity energy-efficient OFDMA," in Proc. IEEE ICC, June 2009, pp. 1-5.

[11] R. G. Gallager, Information Theory and Reliable Communication. John Wiley \& Sons, Inc., 1968.

[12] D. Fudenberg and J. Tirole, Game Theory. MIT Press, 1991.

[13] E. Wolfstetter, Topics in Microeconomics: Industrial Organization, Auctions, and Incentives. Cambridge University Press, 1999.

[14] R. D. Yates, "A framework for uplink power control in cellular radio systems," IEEE J. Sel. Areas Commun., pp. 1341-1347, Sep. 1995.

[15] J. W. Friedman, Oligopoly and the Theory of Games. North-Holland Publishing Co., 1977.

[16] R. G. Bartle, The Elements of Real Analysis. John Wiley, 1964.

[17] S. Boyd and L. Vandenberghe, Convex Optimization. Cambridge University Press, 2004.

[18] P. Viswanath, D. N. C. Tse, and R. Laroia, "Opportunistic beamforming using dumb antennas," IEEE Trans. Inf. Theory., vol. 48, no. 6, pp. 1277-1294, June 2002.

[19] A. T. Koc, S. Talwar, A. Papathanassiou, R. Yang, N. Himayat, and H. Yin, "IEEE c802.16m-08/666r2: uplink power control recommendations for IEEE 802.16m." Available: http://wirelessman.org/tgm/index.html 


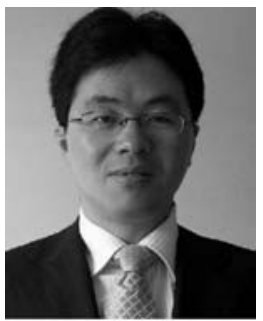

Guowang Miao (S'03, M'10) received a B.S. and a M.S. degree, in 2003 and 2006 respectively, in electronic engineering from Tsinghua University, Beijing, China, and a M.S. degree and a Ph.D. degree, both in 2009, in electrical and computer engineering from Georgia Institute of Technology, Atlanta, GA, USA. Currently he is working at Dallas Telecom Lab of Samsung as a senior algorithms and standard engineer. His research interests are in wireless communications and networking, with a current focus on energy efficient wireless communications, optimization of distributed random access, heterogeneous networks, coordinated multi-point transmission/reception, and PHY-MAC cross-layer design for wireless networks.

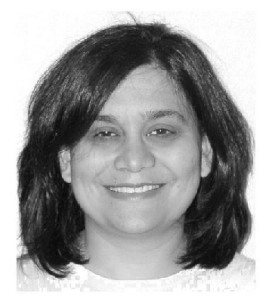

Nageen Himayat (nageen.himayat@intel.com) is a Senior Research Scientist with Intel Labs, where she performs research on several aspects of broadband wireless systems, including multi-tier, heterogeneous networks, cross layer radio resource management, MIMO-OFDM techniques and optimizations for M2M communications. Dr. Himayat has over 15 years of research and development experience in the telecom industry and has held prior appointments with Lucent Technologies and General Instrument Corp, where she developed standards and systems for broadband "last-mile" networks. She obtained her B.S.E.E degree from Rice University, and her Ph.D. degree in Electrical Engineering from the University of Pennsylvania, in 1989 and 1994 respectively.

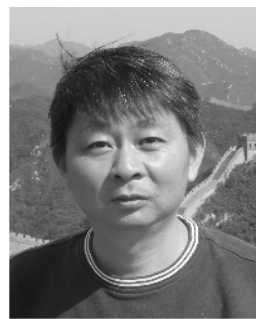

Ye (Geoffrey) Li (S'92, M'95, SM'97, F'06) received his B.S.E. and M.S.E. degrees in 1983 and 1986, respectively, from the Department of Wireless Engineering, Nanjing Institute of Technology, Nanjing, China, and his Ph.D. degree in 1994 from the Department of Electrical Engineering, Auburn University, Alabama. He was a Teaching Assistant and then a Lecturer with Southeast University, Nanjing, China, from 1986 to 1991, a Research and Teaching Assistant with Auburn University, Alabama, from 1991 to 1994, and a Post-Doctoral Research Associate with the University of Maryland at College Park, Maryland, from 1994 to 1996. He was with AT\&T Labs - Research at Red Bank, New Jersey, as a Senior and then a Principal Technical Staff Member from 1996 to 2000. Since 2000, he has been with the School of Electrical and Computer Engineering at Georgia Institute of Technology as an Associate and then a Full Professor. He is also holding the Cheung Kong Scholar title at the University of Electronic Science and Technology of China since March 2006. His general research interests include statistical signal processing and telecommunications, with emphasis on OFDM and MIMO techniques, cross-layer optimization, and signal processing issues in cognitive radios. In these areas, he has published about 200 papers in refereed journals or conferences and two books, 20 of which are with over 100 Google citations. He has over 20 patents granted or filed. He once served or is currently serving as an editor, a member of editorial board, and a guest editor for over 10 technical journals. $\mathrm{He}$ organized and chaired many international conferences, including technical program vice-chair of IEEE ICC'03 and co-chair of IEEE SPARC'11. He has been awarded an IEEE Fellow for his contributions to signal processing for wireless communications since 2006, selected as a Distinguished Lecturer for 2009 - 2010 by IEEE Communications Society, and won 2010 IEEE Communications Society Stephen O. Rice Prize Paper Award in the field of communications theory.

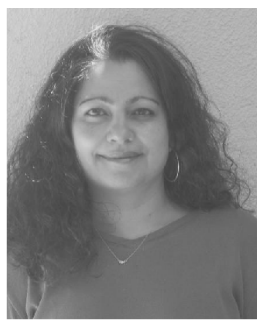

Shilpa Talwar (shilpa.talwar@intel.com) is a Principal Engineer in the Wireless Communications Laboratory at Intel, where she is conducting research on mobile broadband technologies for increasing cellular capacity and coverage. While at Intel, she has led projects on several topics, including advanced MIMO and interference mitigation techniques. Currently, her research is focused on Heterogeneous networks and M2M air interface optimizations. Prior to Intel, she held several senior technical positions in wireless industry. She has over 15 years of experience in wireless. She graduated from Stanford University in 1996 with a Ph.D. in Applied mathematics and an M.S. in electrical engineering. She is the author of numerous technical publications and patents. 